\title{
Illustration de la valeur indicatrice des caractéristiques biologiques des macroinvertébrés d'une communauté benthique à différentes échelles d'observation
}

\author{
P. Usseglio-Polatera ${ }^{1,2}$ \\ S. Thomas ${ }^{2}$ \\ J.-N. Beisel ${ }^{2}$ \\ J.-C. Moreteau ${ }^{2}$
}

Mots-clés : macroinvertébrés benthiques, stratégies écologiques, traits biologiques, mésohabitat, rivière, analyse de co-inertie.

La faune d'un hydrosystème intègre la variabilité spatio-temporelle de l'environnement. Les espèces présentant les caractéristiques biologiques et les stratégies écologiques les plus appropriées à chaque type d'habitat de la mosaïque fluviale sont supposées sélectionnées. Certaines combinaisons d'adaptations sont donc susceptibles d'être plus particulièrement rencontrées dans certains habitats, compte-tenu de leurs caractéristiques environnementales. Par conséquent, une utilisation pertinente des informations autécologiques connues sur les différentes unités systématiques d'un peuplement, doit permettre la restitution et le suivi de certaines des caractéristiques environnementales d'un biotope à travers l'évolution qualitative et quantitative de son peuplement faunistique.

Les mésohabitats les plus représentatifs de douze stations situées sur des cours d'eau d'ordre 1 à 4 appartenant à trois bassins versants du Nord-Est de la France et couvrant un large éventail de situations mésologiques ont été échantillonnés au filet-Surber au printemps et à l'automne 1993 et 1994. Les caractéristiques biologiques et les stratégies écologiques des organismes aquatiques rencontrés sont décrites par 13 variables s'exprimant selon différentes modalités.

La confrontation des connaissances acquises sur l'autoécologie des taxons et de la dynamique spatio-temporelle du peuplement autochtone génère une information claire sur l'organisation et le fonctionnement de la communauté benthique, les caractéristiques de l'environnement et sur leurs modifications éventuelles, à différentes échelles spatiales (mésohabitat, station, bassin...). A chaque type de mésohabitat correspond une communauté benthique qui présente une combinaison originale de traits biologiques et de stratégies écologiques. Les taxons de cette communauté peuvent alors restituer une information pertinente sur les propriétés des mésohabitats qu'ils colonisent, que ce soit en terme de stabilité, de diversité et/ou de potentialités trophiques des niches écologiques proposées.

\section{Biological trait structure of macroinvertebrate benthic communities}

Keywords : benthic macroinvertebrates, ecological strategy, biological traits, mesohabitat, river, co-inertia analysis.

The faunal assemblage of a river ecosystem integrates the spatial and temporal variability of the habitat. Taxa with appropriate combinations of adaptations, concerning especially traits related to survival and reproduction use particular habitat types. Consequently, the synthesis of published autecological information on macroinvertebrate taxa can be used to demonstrate some of the environmental changes of an ecosystem.

Mesohabitats were sampled in spring and autumn 1993 and 1994 in twelve study sites on 1st to 4th order streams in three river catchments located in the northeast of France. Sites were selected to exhibit high microscale heterogeneity.

Affinities of taxa for modalities of biological traits and/or ecological strategies were described. The investigation of the relationship between traits and distributions of taxa can demonstrate some of the characteristics of both faunal community and environment and provide clear information on changes in biotopes across different spatial scales (mesohabitat, station, catchment basin). The benthic assemblage of each mesohabitat type displayed a specific combination of biological traits. Thus, taxa can provide insights about mesohabitat characteristics in terms of stability, diversity or trophic potentialities of their potential ecological niches.

1. ESA 5023 CNRS, Ecologie des Eaux Douces et des Grands Fleuves, 43 Bd du 11 Novembre 1918, F-69622 Villeurbanne Cedex, France

2. Centre de Recherches Ecologiques de l'Université de Metz, B.P. 4116, 1 Rue des Récollets, F-57040 Metz Cedex 01, France.

E-mail : usseglio@sciences.univ-metz.fr 


\section{Introduction}

L'appréhension et la modélisation des relations entre les caractéristiques biologiques des organismes vivants et les variations des facteurs environnementaux dans un écosystème, puis l'utilisation de ces modèles dans la justification des distributions d'abondance des différents taxons de la biocénose autochtone, ont toujours constitué l'un des axes de recherche fondamentaux de l'écologie scientifique (Resh et al. 1994). En effet, la faune d'un écosystème perçoit la variabilité spatiotemporelle de l'environnement de sorte que les espèces présentant les caractéristiques biologiques et les stratégies écologiques les plus appropriées à chaque type d'habitat de la mosaïque benthique, sont supposées sélectionnées (Hildrew \& Edington 1979, Southwood 1988, Ladle \& Ladle 1992, Townsend \& Hildrew 1994). Certaines combinaisons d'adaptations, concernant plus particulièrement les stratégies de reproduction (fécondité, voltinisme, mode d'essaimage), de nutrition (régime alimentaire, mode d'alimentation) et/ou d'occupation de l'espace (affinités pour différents types de substrats ou différentes classes de vitesse de courant), sont donc susceptibles d'être plus particulièrement rencontrées dans certains habitats, compte tenu de leurs caractéristiques environnementales (nature et stabilité du substrat, potentialités trophiques...). Si la dynamique des peuplements aquatiques est considérée comme principalement gouvernée par des processus autécologiques (Townsend \& Hildrew 1994), elle devient alors prédictible à partir de la connaissance des caractéristiques biologiques et des stratégies écologiques développées par la communauté faunistique dans un espace de variabilité spatio-temporelle. Corrélativement, des relevés faunistiques, par la valeur indicatrice de leur peuplement, peuvent fournir des informations sur les caractéristiques mésologiques et fonctionnelles du système étudié.

L'objectif est alors de déterminer si la valeur indicatrice des caractéristiques biologiques des macroinvertébrés d'une communauté benthique, déjà démontrée à l'échelle d'un bassin, ou d'une station (Statzner et al. 1994), reste pertinente à l'échelle du mésohabitat; un mésohabitat étant défini comme l'association originale d'un type de substrat, d'une gamme de vitesses de courant et d'une gamme de hauteurs d'eau.

Usseglio-Polatera (1991, 1994, 1997), Bournaud et al. (1992), Dolédec \& Statzner (1994), Townsend et al. (1997), notamment, ont montré comment utiliser les connaissances bibliographiques acquises sur les caractéristiques biologiques et les stratégies écologiques des différentes espèces d'une communauté pour restituer clairement certaines caractéristiques de l'habitat. La technique du «codage flou» (Chevenet et al. 1994), qui consiste à attribuer à chaque unité systématique de la communauté biocénotique, une note d'affinité pour les différentes modalités de variables biologiques ou écologiques pertinentes permet de structurer l'information écologique de façon appropriée en générant, pour chaque taxon de la communauté, une série de profils biologiques. L'analyse de la structure commune du tableau récapitulant les connaissances acquises sur l'autécologie des taxons réunies sous forme de profils biologiques [tableau : $\mathrm{n}$ taxons $\mathrm{x} \mathrm{p}$ variables distribuées en $\mathrm{m}_{\mathrm{p}}$ modalités] et du tableau décrivant la dynamique spatio-temporelle du peuplement autochtone [tableau : $\mathrm{n}$ taxons $\mathrm{x} \mathrm{r}$ relevés] peut alors répondre de manière efficace à la problématique exposée.

Cette approche est illustrée par la confrontation des traits biologiques ou écologiques et de la structure faunistique du peuplement macrobenthique de 352 relevés de mésohabitats de rivières du nord-est de la France.

\section{Matériel et méthodes}

Le peuplement faunistique des mésohabitats les plus représentatifs de douze stations situées sur des cours d'eau d'ordre 1 à 4 appartenant à trois bassins versants (Haute-Meuse, Mortagne, Bruche) du nord-est de la France (Fig. 1a) et couvrant un large éventail de situations mésologiques, a été échantillonné au filet Surber (surface : $0,05 \mathrm{~m}^{2}$; vide de maille : $300 \mu \mathrm{m}$ ) au printemps et à l'automne 1993 et 1994. Par station et par campagne, huit à douze échantillons ont été prélevés, fixés sur le terrain, puis triés et identifiés au niveau systématique de la famille.

Les caractéristiques biologiques et les stratégies écologiques des organismes aquatiques rencontrés sont décrites par 13 variables qualitatives ou semi-quantitatives qui s'expriment en différentes modalités (Tableau 1). L'affinité d'une espèce pour les différentes modalités d'une variable est alors décrite, selon la technique du «codage flou» (Chevenet et al. 1994), par une série de notes de 0 - aucune affinité de l'espèce pour la modalité considérée - à 5 - l'espèce est très étroitement liée à cette modalité -. Un ensemble de 13 profils biologiques est ainsi obtenu pour chaque unité taxonomique du peuplement [base de données dans Tachet et al. (à paraître)].

Compte-tenu de la complexité des relations entre les caractéristiques biologiques ou écologiques et la structure des peuplements, une approche multivariée est requise (Fig. 1b). Dans un premier temps, le tableau des profils biologiques (notes d'affinité pour les modalités des variables exprimées en distribution de fréquences) 


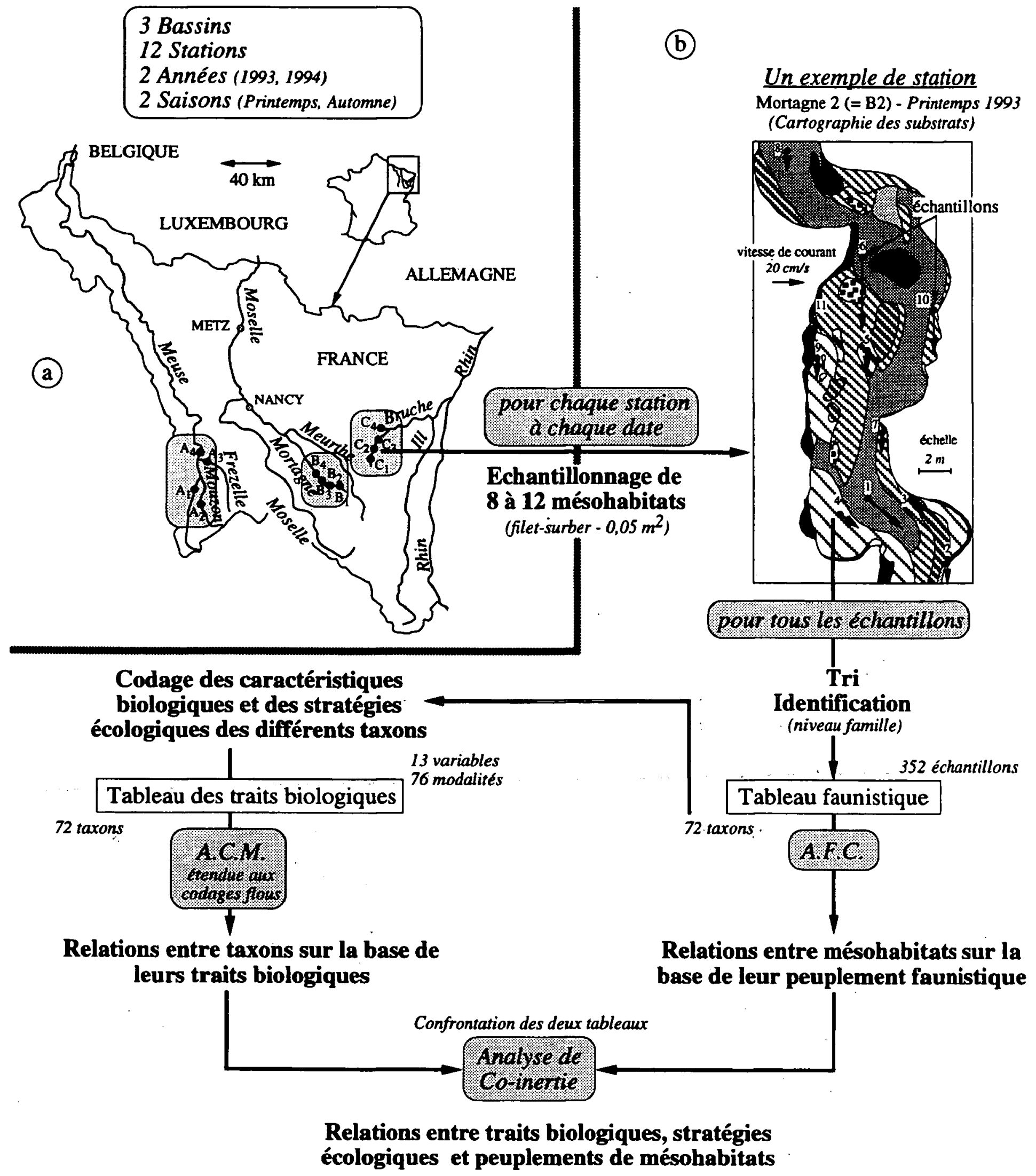

Fig. 1 a. Localisation des stations d'étude. b. Démarche expérimentale et procédure analytique.

Fig. 1 a. Location of the study sites. b. Design of the research strategy and analytical method. 
Tableau 1. Tableau des variables descriptives des caractéristiques biologiques et des stratégies écologiques des 72 unités taxonomiques retenues pour l'analyse. Modalités et codes utilisés dans les figures.

Table 1. Biological traits and modalities (= categories, plus their codes used as labels in the figures) of 72 taxa used in this analysis.

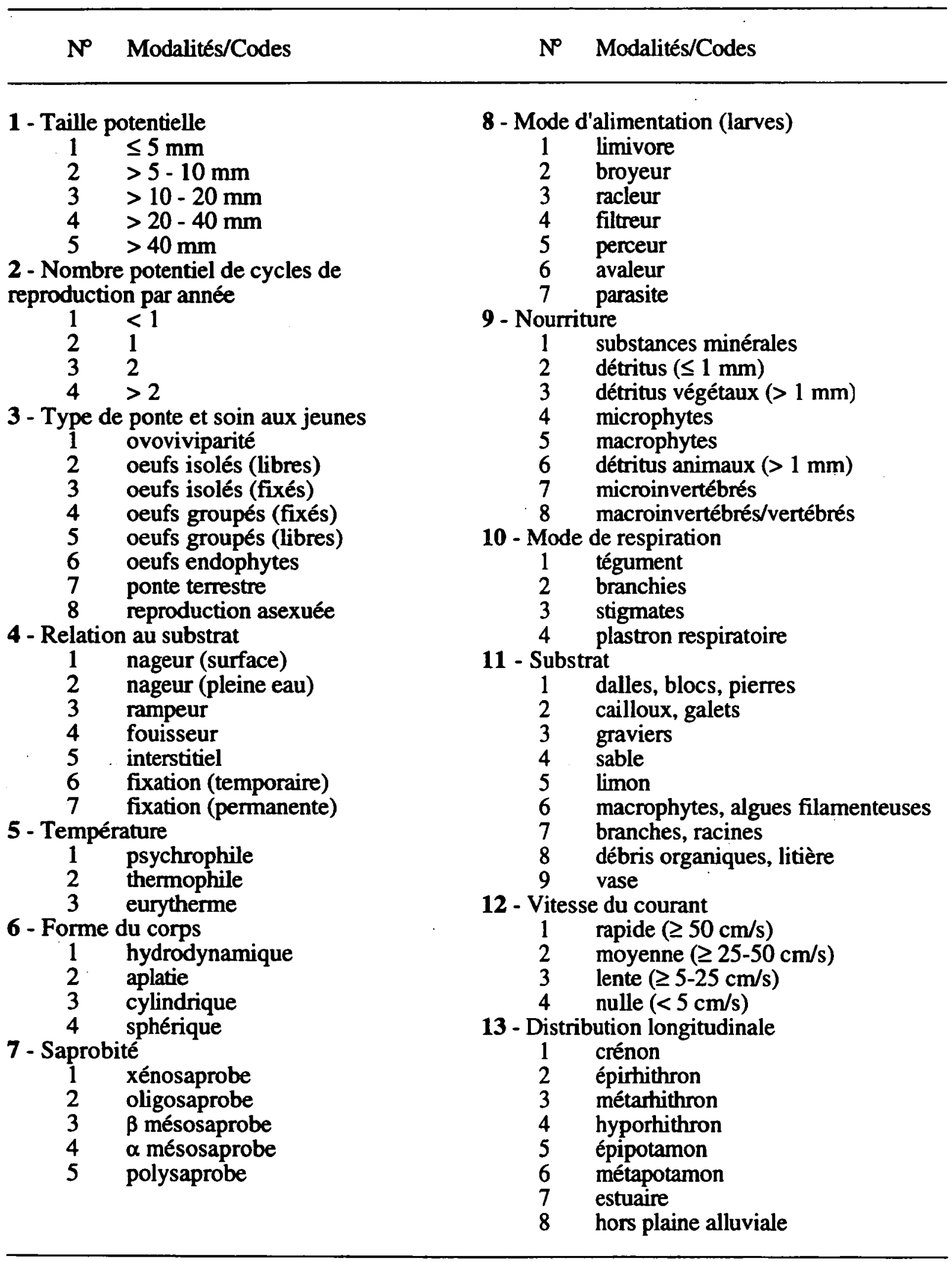


et le tableau décrivant la dynamique spatio-temporelle des peuplements benthiques (abondances faunistiques exprimées en logarithme de base 2 pour 72 familles présentes dans plus de trois relevés) sont respectivement traités par Analyse des Correspondances Multiples sur «codages flous» (Chevenet et al. 1994) et par Analyse Factorielle des Correspondances de manière indépendante (résultats non développés dans cet article). Puis, une analyse de la co-inertie du couple de tableaux (Dolédec \& Chessel 1994) est utilisée pour décrire les relations entre traits biologiques et dynamique des peuplements. Les calculs et représentations graphiques ont été réalisés avec le logiciel ADE 4.0 (Thioulouse et al. 1995).

\section{Résultats}

L'analyse de co-inertie recherche le meilleur compromis alliant corrélation et structure du tableau des profils biologiques et du tableau de structure faunistique. Les bonnes corrélations obtenues entre les axes factoriels $F 1(0,825)$ et les axes factoriels F2 $(0,644)$ tendent à montrer qu'une bonne partie des structures des deux tableaux sont en correspondance. Les macroinvertébrés ayant des profils biologiques particuliers, présentent donc des distributions spatio-temporelles caractéristiques. L'examen simultané des positions des relevés faunistiques (Fig. 2), des modalités des 13 variables (Fig. 3) et de la représentation vectorielle des taxons (Fig. 4) sur le plan factoriel F1-F2 permet de préciser les résultats obtenus.

Les relevés faunistiques sont clairement ordonnés suivant l'axe principal, en fonction de leur appartenance à un bassin hydrographique (Fig. 2a). Le peuplement macrobenthique des stations des bassins de la Bruche et de la Mortagne se caractérise en effet par une certaine homogénéité de la structure faunistique et des caractéristiques biologiques. Il se démarque nettement du peuplement des stations du bassin de la Haute-Meuse [Meuse à Bazoilles (Station A1; Fig. 1a) ou à Brixey (Station A4; Fig. 1a ) et Mouzon suivant l'axe F1; Frézelle suivant l'axe F2 - Fig. 2b] qui, par ailleurs, manifeste une plus forte hétérogénéité. Les différences de structure faunistique observées sont à associer à une différence dans la composition de la mosaïque d'habitats caractéristique de chaque groupe de stations (Fig. 2c), avec une contribution sans doute plus importante des habitats correspondant à des substrats de fine granulométrie (Meuse à Brixey, Meuse à Bazoilles et Mouzon) ou des débris organiques (Frézelle), quelle que soit la saison (Fig. 2c), à la composition du peuplement faunistique des stations du bassin de la Meuse.
Le peuplement des stations de la Mortagne et de la Bruche présente un cortège d'espèces semi- ou univoltines (var. 2 - Fig. 3), plutôt caractéristiques du rhithron (var. 13), qui manifestent une affinité plus forte pour les substrats de granulométrie grossière (blocs, cailloux, graviers - var. 11) et une tendance plus nette à la rhéophilie (var. 6 et 12) et à la psychrophilie (var. 5). Sensibles à une contamination organique de leur biotope (var. 7), les espèces, plutôt racleuses de substrat (var. 8), exploitent principalement les microphytes (var. 9). Les Plécoptères dans leur ensemble (Fig. 4a), les Trichoptères Goeridae, Glossosomatidae, Rhyacophilidae et Brachycentridae (Fig. 4b), les Ephéméroptères Heptageniidae et Ephemerellidae (Fig. 4c), les Coléoptères Elmidae (Fig. 4d), les Diptères Blephariceridae (Fig. 4f) et les Gastéropodes Ancylidae (Fig. 4h) sont des éléments caractéristiques de ce peuplement. Il s'oppose au peuplement des stations du bassin de la Haute-Meuse composé de taxons, à temps de génération plus court (var. 2), aux caractères limnophiles plus affirmés (var. 4,$6 ; 11$ et 12 ), eurythermes voire thermophiles (var. 5), plutôt broyeurs (Frézelle) ou mangeurs de fins dépôts (autres stations), prédateurs ou exploitant principalement les débris organiques (var. 8 et 9). La communauté faunistique correspondante est particulièrement bien représentée par les Trichoptères Hydroptilidae, Leptoceridae et Polycentropodidae, les Ephéméroptères Caenidae et Ephemeridae, les Coléoptères Haliplidae, Hydrophilidae et Dytiscidae, les Odonates, les Hétéroptères, les Mégaloptères et la plupart des familles de Diptères, de Mollusques et de Crustacés (Fig. 4).

L'adéquation entre caractéristiques biologiques et distribution spatio-temporelle d'une famille systématique est d'autant meilleure que sa représentation vectorielle sur le plan F1-F2 est plus courte (Fig. 4). Il existe, par exemple, une bonne correspondance entre les traits biologiques et la distribution d'abondances dans les trois bassins étudiés de la plupart des familles de Diptères (Stratiomyidae et Blephariceridae exceptés). Ce qui n'est pas le cas des Odonates ou des Hétéroptères. La figure 4 illustre, par ailleurs, l'homogénéité de caractéristiques biologiques et de la distribution spatio-temporelle des Plécoptères (les origines d'une part, et les extrémités d'autre part, des vecteurs représentatifs des différentes familles sont situées dans deux zones restreintes du plan factoriel). Les Trichoptères et les Ephéméroptères des stations prospectées présentent une plus grande hétérogénéité de traits biologiques et de distribution. Les Mollusques Gastéropodes et Bivalves, qui se caractérisent par une combinaison de traits biologiques notablement différente (les vecteurs caractérisant les familles de ces deux groupes systéma- 

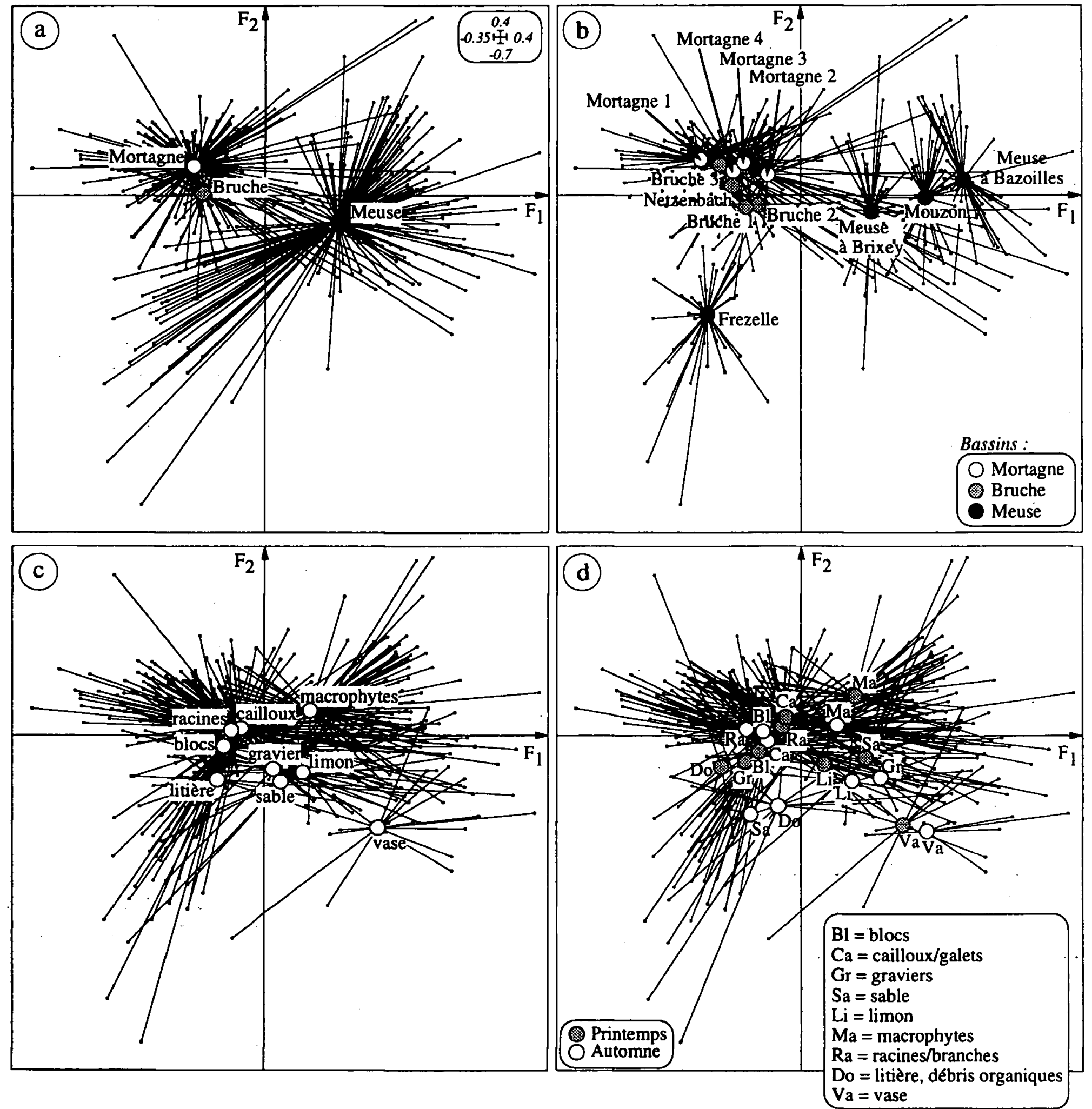

Fig. 2. Co-structure entre caractéristiques biologiques et distributions d'abondance de 72 taxons de macroinvertébrés, observée par analyse de co-inertie. Carte factorielle F1 x F2 des relevés faunistiques du tableau de distribution d'abondances. Les trois bassins versants (a) sont positionnés (cercles) à la moyenne pondérée des relevés (petits carrés noirs) qui leur correspondent. Un trait relie chacun des relevés à la position moyenne du bassin auquel il appartient. Les 12 stations (b) et les 9 types de substrat (c) toutes saisons confondues et les 9 types de substrat, compte-tenu de la saison à laquelle le relevé a été effectué (d) sont positionnés suivant le même principe.

Fig. 2. Co-structure between biological traits and distributions of 72 macroinvertebrate taxa by co-inertia analysis. Distribution of faunal samples (small squares) on the F1 x F2 plane. (a) Ordination of three catchment basins (circles). Each basin is positioned at the weighted average of samples performed in that basin. Lines link samples to basins. Ordination of twelve stations and nine substrate types, all dates pooled (b \& c) and nine substrate types according to their sampling date (d) on the F1 x F2 plane, using the same procedure. 

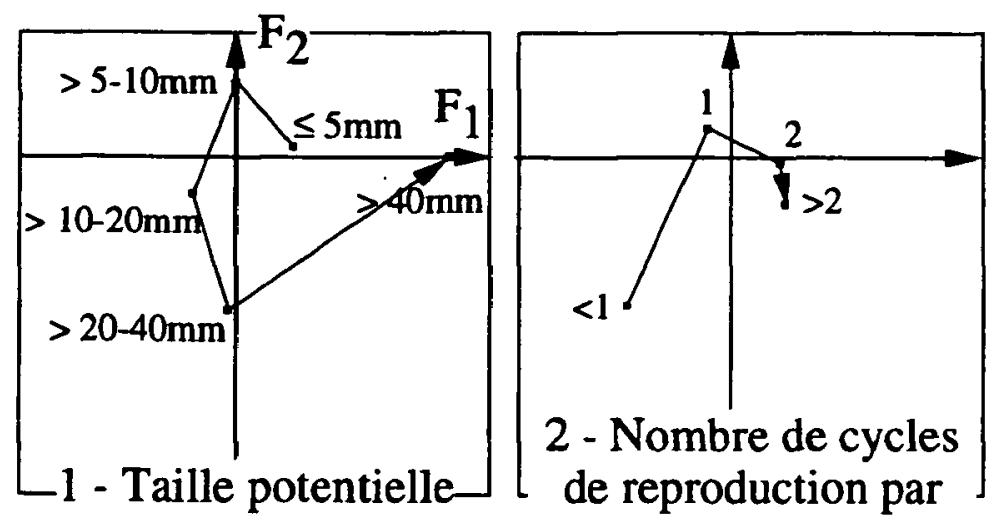

2 - Nombre de cycles de reproduction par année
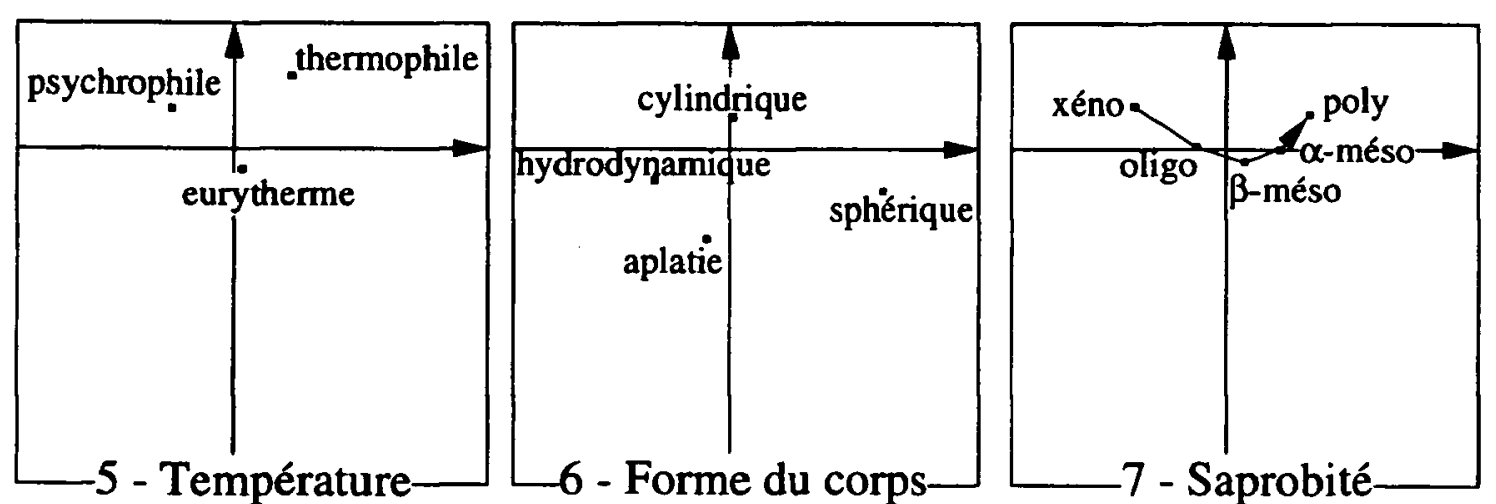

7 - Saprobité-

-11 - Substrat

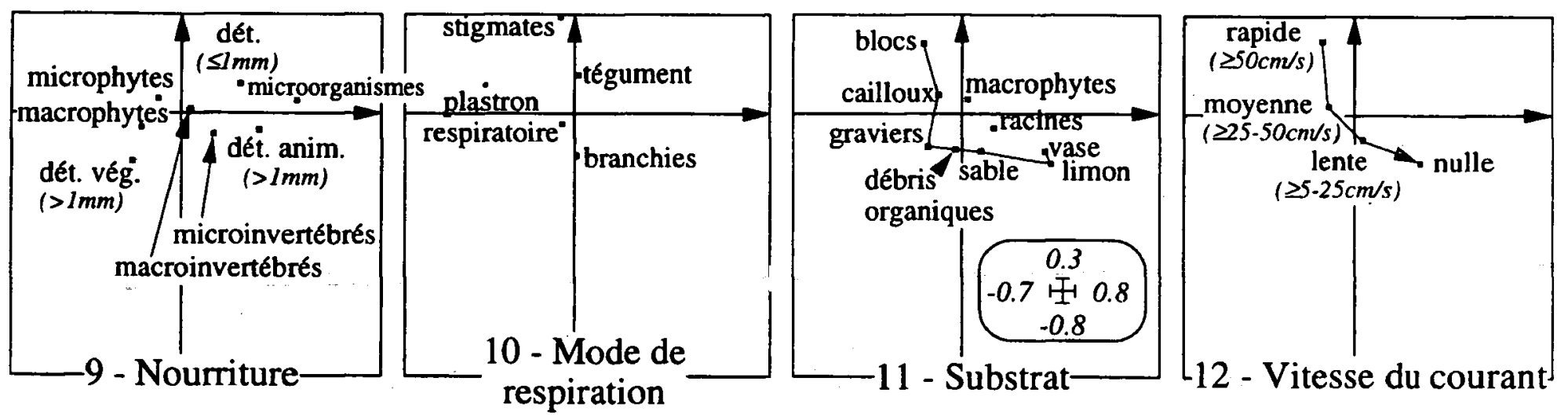

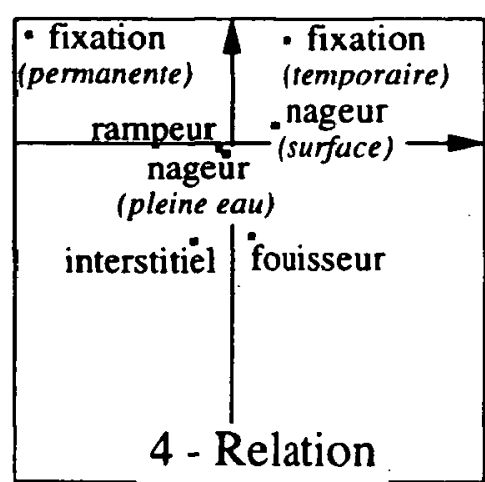

au substrat

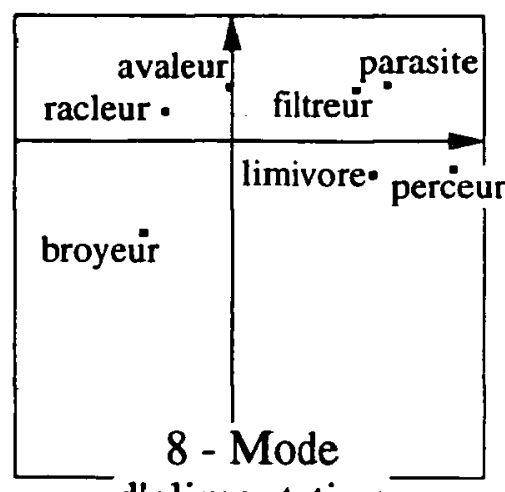

d'alimentation

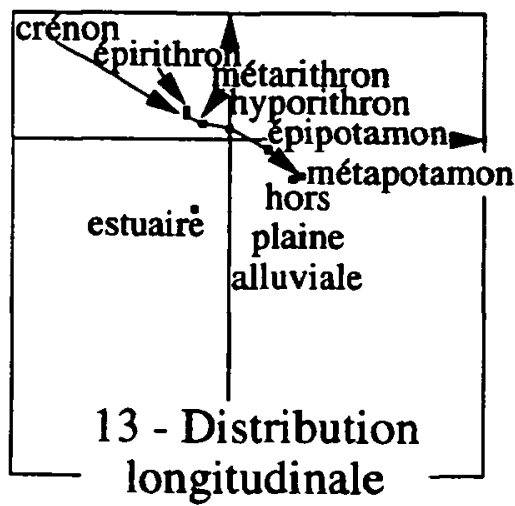

Fig. 3. Modalités des 13 variables biologiques et écologiques dans le plan F1-F2 de l'analyse de co-inertie. Les codes descriptifs des modalités sont ceux du Tableau 1.

Fig. 3. Ordination of 13 biological and ecological variables on the F1 x F2 plane of the co-inertia analysis. See Table 1 for full labels of traits and modalities. 


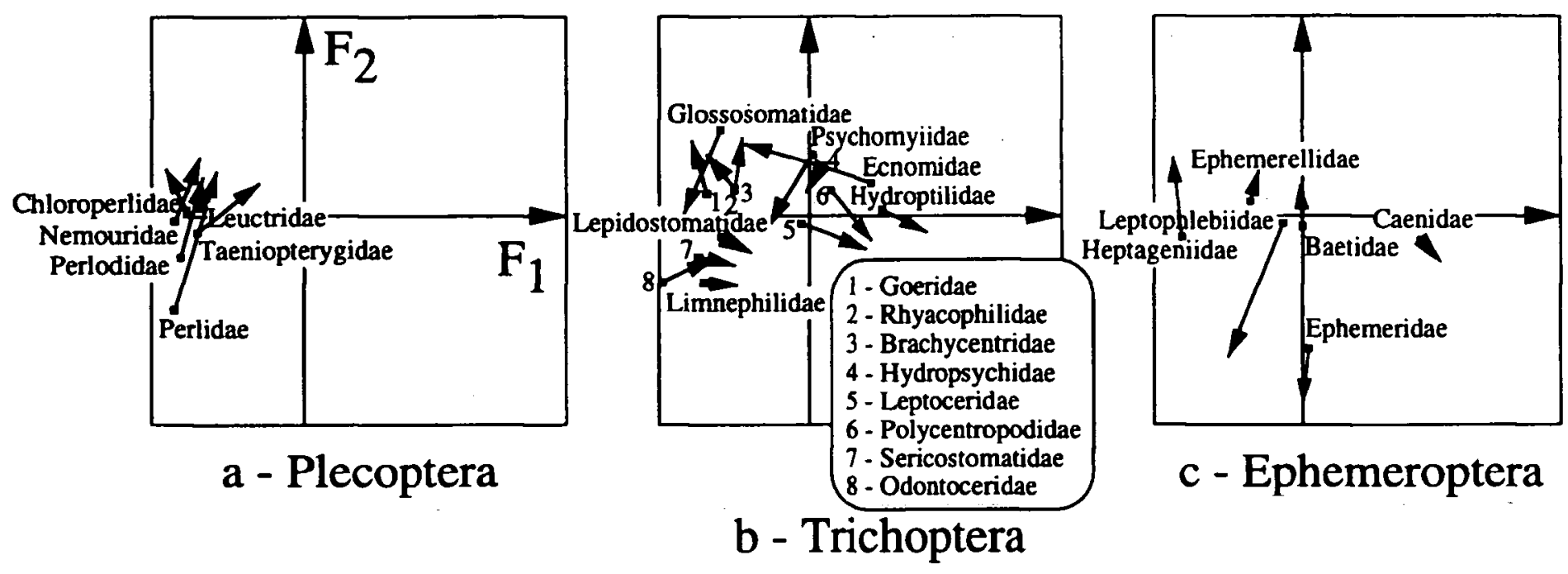

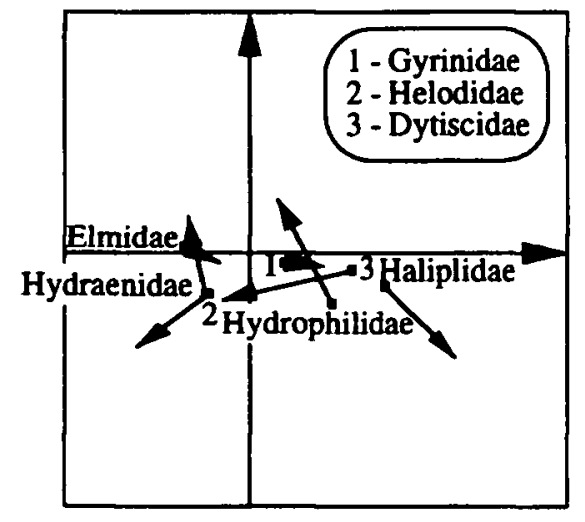

d - Coleoptera

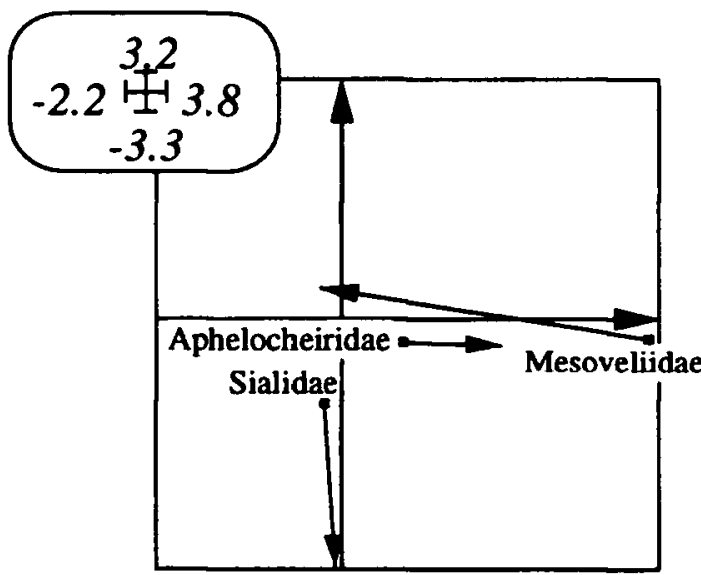

g - Megaloptera Heteroptera

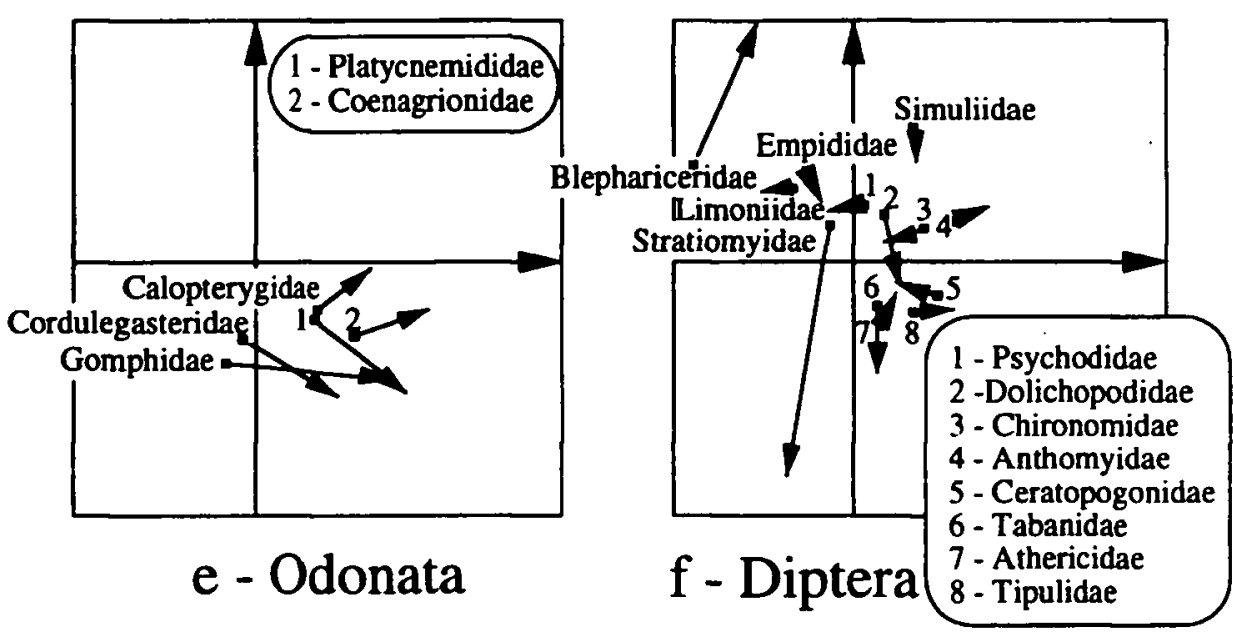

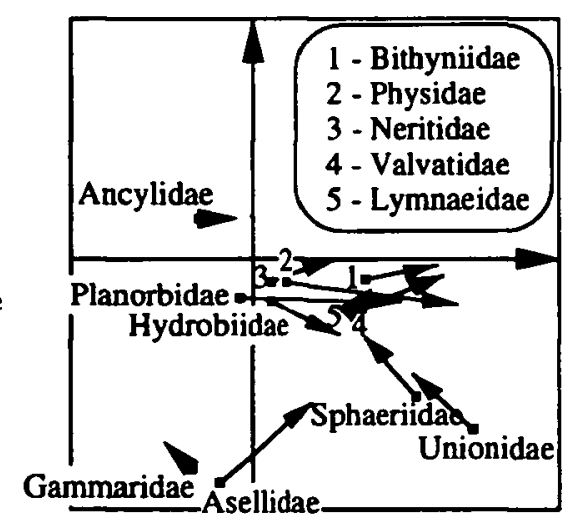

h - Gastropoda Bivalvia Crustacea

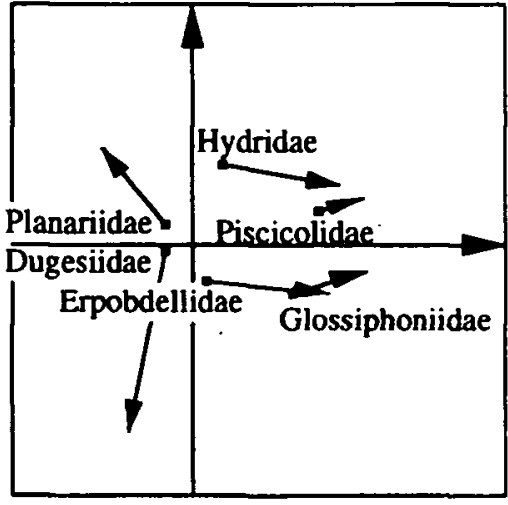

i - Cnidaria

Turbellaria Achaeta

Fig. 4. Confrontation des coordonnées des taxons sur les plans F1-F2 de l'analyse de co-inertie. Chaque taxon est représenté par un vecteur. L'origine de chaque vecteur est positionnée par la coordonnée du taxon associée au tableau des traits biologiques et écologiques, l'extrémité par la coordonnée associée au tableau des distributions d'abondances. Les variances sont unitaires sur chaque axe pour ramener les deux systèmes à une même échelle.

Fig. 4. Comparison of taxa positions on the two F1 x F2 planes by co-inertia analysis. Each taxon is defined by an arrow. The beginning of the arrow marks the position according to the biological and ecological traits of a taxon and the end of the arrow marks its position according to the habitat utilization. Biological and faunal scores are standardized. 
tiques ont une origine située dans deux zones nettement différentes du plan factoriel) ont par contre une distribution spatio-temporelle très semblable (les deux groupes de vecteurs sont convergents). Inversement, les Planariidae et les Dugesiidae, aux caractéristiques biologiques voisines (origine voisine des deux vecteurs), ont des répartitions spatio-temporelles nettement différentes (vecteurs divergents).

\section{Discussion}

La distribution spatio-temporelle des macroinvertébrés benthiques dans une portion de cours d'eau peut être considérée comme le résultat d'un ensemblé d'interactions, entre variables biotiques ou abiotiques du type vitesse du courant, substrat, profondeur, ressources trophiques et/ou compétition. Les variations observées dans la structure des communautés faunistiques dépendront donc plus ou moins directement de modifications de ces paramètres, à différentes échelles spatiales (inter-bassins, inter-stationnelle, inter-habitats) ou temporelles (inter-saisonnière, inter-annuelle).

Les positions moyennes, nettement différentes, des relevés réalisés sur des substrats de même nature (Fig. 2c), tendent à montrer qu'à chaque type de mésohabitat correspond une communauté benthique qui présente une combinaison de traits biologiques et de stratégies écologiques spécifiques.

La projection des coordonnées des substrats minéraux sur l'axe F1 permet de considérer cet axe comme décrivant, en particulier, un gradient décroissant de granulométrie des blocs vers les limons/vases. Les substrats de granulométrie grossière sont préférentiellement colonisés par des organismes morphologiquement adaptés (forme hydrodynamique), qui, dans le cadre du modèle r-K de stratégies biologiques - au sens de McArthur \& Wilson (1963) ou Pianka (1970) -, réuniraient plutôt une combinaison de traits proches de l'extrémité $\mathrm{K}$ du gradient (cycle long, taille moyenne, pontes fixées), et seraient les plus exigeants quant à la qualité de l'habitat (rhéophilie, psychrophilie, xénoou oligo-saprobie).

Les substrats minéraux de fine granulométrie sont colonisés par des taxons, plutôt assimilables à des stratèges de type $r$ (polyvoltinisme, oeufs le plus souvent libérés au hasard dans le milieu). Certains des traits biologiques observés ne correspondent pas cependant aux caractères attendus. Les taxons peuvent en effet être de petite taille $(<0,5 \mathrm{~cm})$ mais également avoir une taille importante $(\geq 4 \mathrm{~cm})$. C'est le cas des larves d'Odonates présentes dans ce type d'habitats. Les caractéristiques biologiques des organismes d'un peuple- ment de chevelu racinaire s'apparentent plutôt aux caractéristiques des communautés faunistiques observées sur les substrats minéraux de granulométrie grossière. Le peuplement faunistique des litières et débris organiques est paradoxalement plus proche (en particulier suivant F1) du peuplement faunistique des substrats d'érosion que des substrats de sédimentation. La position moyenne des macrophytes est assez délicate à interpréter, dans la mesure où sous l'appellation «macrophytes», est réuni un ensemble hétérogène d'habitats de structure et d'architecture notablement différente (bryophytes, hélophytes, hydrophytes, algues filamenteuses).

Les variations saisonnières (Fig. 2d) ou interannuelles (résultats non présentés) dans la composition faunistique moyenne du peuplement des différents types d'habitats sont souvent faibles. Lorsqu'elle est avérée, elle concerne des habitats minéraux de faible granulométrie (sable, gravier), mais ceci de manière non systématique (cf. la position moyenne des vases et des limons au printemps et à l'automne - Fig. 2d).

Les différences de traits biologiques et de stratégies écologiques entre communautés faunistiques résultent donc de différences dans les caractéristiques mésologiques des habitats qu'elles colonisent. Il est à noter la proximité des positions des 9 modalités de la variable «substrat» dans le plan F1-F2, définies (1) d'une part par les notes d'affinités attribuées, sur la base d'informations bibliographiques, aux différentes unités systématiques rencontrées lors de la campagne d'échantillonnage (var. 4 - Fig. 3), (2) en recalculant, d'autre part, la position moyenne des relevés réalisés sur chaque substrat-type, compte-tenu de leurs distributions d'abondances respectives (Fig. 2c).

L'effet station, dépend évidemment de la structure et de la composition de la mosaïque d'habitats offerte aux macroinvertébrés. Cet effet inter-stationnel démarque nettement les stations du bassin versant de la Haute-Meuse. Appartenant typiquement à des rivières de plaine, ces stations présentent un peuplement faunistique plutôt bien adapté à l'instabilité environnementale (stratèges $r$ dominants). Elles s'opposent en celà aux stations de piémont des bassins de la Bruche et de la Mortagne. La Meuse à Bazoilles, à Brixey et le Mouzon se caractérisent par des peuplements faunistiques qui présentent de fortes affinités, à la fois en termes de distributions d'abondances et de traits biologiques, sans doute parce qu'elles offrent certaines caractéristiques mésologiques communes, en particulier une forte végétalisation (Beisel 1996). La Frézelle, présente, sur le plan faunistique, une situation intermédiaire entre d'une part, les stations de la Meuse et du 
Mouzon et d'autre part, les stations du massif vosgien. Il semble d'ailleurs que, malgré sa position géographique, la Frézelle ait plus de points communs avec ces dernières qu'avec les stations de Haute-Meuse (Beisel 1996).

L'expression graphique de la signification biologique et écologique d'un peuplement faunistique, fondamentalement basée sur une revue bibliographique des connaissances sur l'autécologie de ses différentes familles, permet donc d'identifier des combinaisons caractéristiques de traits biologiques et de stratégies écologiques dans les peuplements des différents habitats d'une mosaique fluviale. Ces combinaisons de traits sont (1) d'une part, à mettre en relation avec certaines des caractéristiques mésologiques des habitats colonisés et (2) d'autre part, capables de restituer une information claire sur certaines propriétés des mosaïques benthiques d'habitats, qui réfèrent en particulier à la stabilité et à la diversité des niches écologiques offertes. A l'échelle de la station ou du bassin versant, l'image restituée de la biocénose, en termes de caractéristiques biologiques et écologiques dépend fondamentalement de la structure et de la composition en mésohabitats de la mosaïque benthique.

\section{Travaux cités}

Beisel J.-N. 1996. - Microrépartition des invertébrés benthiques en eau courante : caractéristiques des microhabitats et organisation de leurs peuplements. Thèse de doctorat, Université de Metz : $242 \mathrm{p}$.

Bournaud M., Richoux P. \& Usseglio-Polatera P. 1992. - An approach to the synthesis of qualitative ecological information : the example of aquatic Coleoptera. Regulated Rivers: Research \& Management, 7 : 165-180.

Chevenet F., Dolédec S. \& Chessel D. 1994. - A fuzzy coding approach for the analysis of long-term ecological data. Freshwater Biol., 31 : 295-309.

Dolédec S. \& Chessel D. 1994. - Co-inertia analysis: an alternative method for studying species-environment relationships. Freshwater Biol., 31 : 277-294.
Dolédec S. \& Statzner B. 1994. - Theoretical habitat templets, species traits, and species richness: 548 plant and animal species in the Upper Rhône River and its floodplain. Freshwater Biol., 31 : 523-538.

Hildrew A.G. \& Edington J.M. 1979. - Factors facilitating the coexistence of hydropsychid caddis larvae (Trichoptera) in the same river system. J. Anim. Ecol., 48 : 557-576.

Ladle M. \& Ladle R.J. 1992. — Life history patterns of river invertebrates. Hydrobiologia, $248: 31-37$.

McArthur R.H. \& Wilson E.O. 1963. - An equilibrium theory of insular zoogeography. Evolution, $17: 373-387$.

Pianka E.R. 1970. - On r- and K-selection. Am. Nat., 104 : 592-597.

Resh V.H., Hildrew A.G., Statzner B. \& Townsend C.R. 1994. Theoretical habitat templets, species traits, and species richness: a synthesis of long-term ecological research on the Upper Rhône River in the context of concurrently developed ecological theory. Freshwater Biol., 31 : 539-554.

Southwood T.R.E. 1988. - Tactics, strategies and templets. Oikos, $52: 3-18$.

Statzner B., Resh V.H. \& Dolédec S. (eds) 1994. - Ecology of the Upper Rhône River: a test of habitat templet theories. Freshwater Biol., Special Issue, 31 : 253-556.

Tachet H., Richoux P., Usseglio-Polatera P. \& Bournaud M. (à paraître) - Introduction à l'étude des macroinvertébrés des eaux douces. Approches systématique et écologique.

Thioulouse J., Dolédec, S., Chessel, D. \& Olivier J.M. 1995. ADE software: multivariate analysis and graphical display of environmental data. in Software per l'Ambiente, Guariso G. \& A. Rizzoli'(eds), Bologna : 57-62.

Townsend C.R., Dolédec S. \& Scarsbrook M.R. 1997. - Species traits in relation to temporal and spatial heterogeneity in streams: a test of habitat templet theory. Freshwater Biol., $37:$ 367-387.

Townsend C.R. \& Hildrew A.G. 1994. - Species traits in relation to a habitat templet for river systems. Freshwater Biol., 31: 265-275.

Usseglio-Polatera P. 1991. - Représentation graphique synthétique de la signification écologique d'un peuplement. Application aux macroinvertébrés du Rhône à Lyon. Bull. Ecol., 22 : 195-202.

Usseglio-Polatera P. 1994. - Theoretical habitat templets, species traits, and species richness: aquatic insects in the Upper Rhône River and its floodplain. Freshwater Biol., 31 : 417-437.

Usseglio-Polatera P. 1997. — Long-term changes in the Ephemeroptera of the River Rhône at Lyon, France, assessed using a fuzzy coding approach. in Advances in Ephemeroptera Biology, Lausanne, Sartori M. \& P. Landolt (eds), Lausanne : 227-234. 